\title{
Piperine Enhances the Antioxidant and Anti-Inflammatory Activities of Thymoquinone against Microcystin-LR-Induced Hepatotoxicity and Neurotoxicity in Mice
}

\author{
Mohamed M. Abdel-Daim $\mathbb{D D}^{1},{ }^{1}$ Amany A. Sayed, ${ }^{2}$ Ahmed Abdeen $\mathbb{D}^{\mathrm{D}},{ }^{3}$ Lotfi Aleya, \\ Daoud Ali $\oplus^{5},{ }^{5}$ Abdullah A. Alkahtane, ${ }^{5}$ Saud Alarifi, ${ }^{5}$ and Saad Alkahtani ${ }^{5}$ \\ ${ }^{1}$ Pharmacology Department, Faculty of Veterinary Medicine, Suez Canal University, Ismailia 41522, Egypt \\ ${ }^{2}$ Zoology Department, Faculty of Science, Cairo University, Giza 12613, Egypt \\ ${ }^{3}$ Department of Forensic Medicine and Toxicology, Faculty of Veterinary Medicine, Benha University, Toukh 13736, Egypt \\ ${ }^{4}$ Bourgogne Franche-Comté University, Chrono-Environment Laboratory, UMR CNRS 6249, 25030 Besançon Cedex, France \\ ${ }^{5}$ Department of Zoology, Science College, King Saud University, Riyadh, Saudi Arabia
}

Correspondence should be addressed to Mohamed M. Abdel-Daim; abdeldaim.m@vet.suez.edu.eg

Received 6 November 2018; Accepted 18 February 2019; Published 16 April 2019

Guest Editor: Débora V. Valencia

Copyright (c) 2019 Mohamed M. Abdel-Daim et al. This is an open access article distributed under the Creative Commons Attribution License, which permits unrestricted use, distribution, and reproduction in any medium, provided the original work is properly cited.

\begin{abstract}
Microcystin- (MC-) LR is the most frequent cyanotoxin produced by Microcystis aeruginosa cyanobacteria in the contaminated freshwater environment. MC represents a health hazard to humans and animals. Therefore, the present study was designed to evaluate the potential ameliorative effect of thymoquinone (TQ) and/or piperine (PP) against MC toxicity in mice. Fifty-six mice were randomly divided into seven experimental groups. Group I is the normal control that received distilled water for 21 days; Group II (TQ) was treated with TQ (10 mg/kg, i.p) for 21 days; Group III (PP) was treated with PP (25 mg/kg, i.p) for 21 days; Group IV (MC) was treated with MC $(10 \mu \mathrm{g} / \mathrm{kg}$, i.p) for 14 days and served as the toxic control; and Groups V, VI, and VII received TQ and/or PP 7 days prior to MC and continued for 14 days with MC. The results revealed that MC elicited hepatotoxicity and neurotoxicity which was evident due to the significant elevation of serum AST, ALT, $\gamma$ GT, ALP, LDH, IL- $1 \beta$, IL-6, and TNF- $\alpha$ levels. Furthermore, MC markedly increased MDA and NO contents along with reduction of GSH, SOD, CAT, and GSH-Px in liver and brain tissues. The electron transport chain may be a possible target for MC. TQ and/or PP ameliorated the MC-mediated oxidative damage in the liver and brain which might be attributed to their antioxidant properties. However, the concurrent treatment of TQ and PP showed the best regimen as a result of the PP-enhanced bioavailability of TQ.
\end{abstract}

\section{Introduction}

Microcystin- (MC-) LR is the most abundant cyanotoxin released by Microcystis aeruginosa cyanobacteria (bluegreen algae) into freshwater bodies as a result of extensive anthropogenic eutrophication [1]. MC, which is known to withstand higher temperatures (up to $300^{\circ} \mathrm{C}$ ) increasing its persistence in the aquatic ecosystem, together with excessive production lead to the build-up of high concentrations in the environment offering potential sources of exposure for humans, animals, fish, and birds, mainly through consumption of contaminated seafood, vegetables, and drinking water $[2,3] . \mathrm{MC}$ is described to be a potent hepato- and neurotoxin [2, 4-6]. MC poisoning has been recently reported in many countries including China [7], Egypt [8], Japan [9], Poland [10], and Brazil [11, 12]. Additionally, the MC-prolonged exposure promotes oncogenesis in humans [13], together with the abovementioned; MC has become a great global health concern.

MC is known to exert its hepatotoxicity and neurotoxicity via the inhibition of phosphatase enzymes [4]. Through the biliary system, MC reaches the hepatocytes, wherein it has high affinity with the serine/threonine-containing enzyme phosphatases causing hyperphosphorylation and enzyme 
dysfunction. These events disrupt the signaling pathways and cytoskeleton integrity; thus, MC-induced hepatotoxicity occurs $[4,14,15]$. Independent to these mechanisms, several lines of recent evidence reported the involvement of oxidative stress in $\mathrm{MC}$-induced toxicities through the overproduction of reactive oxygen species (ROS) and suppression of the cellular antioxidant system $[6,16]$. However, the exact mechanisms underlying the MC-induced toxicity remain unclear. Therefore, exploring new mechanistic insights, as well as new antioxidative agents, which protects against MCinflicted toxicities would be of great impact on animal and human health.

Recently, attention has been paid to the role of medicinal plants as a source of natural antioxidants. Thymoquinone (TQ) is the main constituent of Nigella sativa (black seed) with antioxidant, anti-inflammatory, and anticarcinogenic activities. Several reports have documented the protective effect of TQ against the oxidative damage inflicted by paracetamol [17] and lead [18] in the liver; TQ could also alleviate the inflammation associated with Alzheimer's disease [19].

Piperine (PP) is the bioactive alkaloid ingredient of black pepper (Piper nigrum). PP has various pharmacological potencies including antioxidant and anti-inflammatory actions [20, 21]. Recently, coadministration of plant extracts has gained great attention, whereas using two or more components may enhance their clinical efficacy [22]. Lambert et al. [23] have demonstrated that the bioavailability of (-)-epigallocatechin3 -gallate was increased by 1.7 -fold when it was coadministrated with PP in a mouse model. Despite the proven antioxidant activity of TQ, it has poor bioavailability due to its lower water solubility, poor absorption when given orally, and rapid elimination from plasma [24]. Accordingly, we hypothesize that PP may potentially enhance the antioxidant and antiinflammatory efficacy of TQ by increasing its bioavailability. The present study was designed to comparatively evaluate the therapeutic potential of TQ, PP, and their combination against the MC-induced oxidative damage in hepatic and brain tissue. Liver function tests, proinflammatory cytokines, and oxidative status markers were determined.

\section{Materials and Methods}

2.1. Chemicals. Microcystin- (MC-) LR, thymoquinone (TQ), and piperine (PP) were procured from Sigma-Aldrich (Saint Louis, MO, USA). All kits used for biochemical and oxidant/antioxidant markers analyses were purchased from the Laboratory Biodiagnostics Company (Cairo, Egypt) except the lactate dehydrogenase (LDH) enzyme kit (Randox Laboratories Ltd., UK). Interleukin-1 $\beta$ (IL-1 $\beta$ ) and Interleukin-6 (IL6) were obtained from Glory Science Co. Ltd. (Del Rio, TX, USA) and tumor necrosis factor- $\alpha$ (TNF- $\alpha$ ) was purchased from BioSource International Inc. (Camarillo, CA, USA) to assess the inflammatory responses.

2.2. Animals. Adult male albino mice (Mus musculus) weighing 25-30 g were used in the present study. They were housed and maintained in controlled conditions at $25 \pm 2^{\circ} \mathrm{C}$ with a $12: 12$ light-dark cycle. Food and water were given ad libitum. Before commencement of the experiment, all animals were acclimatized to the laboratory conditions for 7 days.

The experimental protocols and procedures used in this study were approved by the Cairo University, Faculty of Science, Institutional Animal Care and Use Committee, Egypt (CU/I/F/39/18). All the experimental procedures were carried out in accordance with international guidelines for the care and use of laboratory animals.

2.3. Experimental Protocol. Fifty-six mice were equally divided into seven experimental groups. Group I (control): mice received distilled water as a vehicle control only for 21 days. Group II (TQ): mice received thymoquinone $(10 \mathrm{mg} / \mathrm{kg}$ daily, i.p; Talib (2017)) for 21 days. Group III (PP): mice received piperine $(25 \mathrm{mg} / \mathrm{kg}$ daily, i.p; Talib (2017)) for 21 days. Group IV (MC): mice received microcystin-LR $(10 \mu \mathrm{g} / \mathrm{kg}$ daily, i.p) for 14 days and then were administered distilled water daily for the subsequent 7 days and served as the MC-intoxicated group [25]. Group V (MC-TQ): mice received thymoquinone only for 7 days and then were treated with TQ and MC concurrently for the subsequent 14 days. Group VI (MC-PP): mice received piperine only for 7 days and then were treated with PP and MC concurrently for the subsequent14 days. Group VII (MC-TQ-PP): mice were coadministered with TQ and PP for 7 days and then treated with TQ, PP, and MC concurrently for the subsequent 14 days.

2.4. Blood and Tissue Sampling. After $24 \mathrm{~h}$ from the last treatment, all groups were euthanized under sodium pentobarbital overdose and blood samples were collected via heart puncture. The blood sample was centrifuged and the serum collected and stored at $-20^{\circ} \mathrm{C}$ for further determination of liver function tests and proinflammatory cytokines (IL-1 $\beta$, IL-6, and TNF$\alpha)$. The liver and brain were rapidly excised, perfused in physiological saline, and homogenized in ice-cold $0.2 \mathrm{M}$ Tris-HCl buffer ( $\mathrm{pH} 7.4$ ) followed by cooled centrifugation $\left(4^{\circ} \mathrm{C}\right)$ at $10000 \mathrm{xg}$. Then, the resultant supernatant was collected and stored at $-80^{\circ} \mathrm{C}$ for determination of tissue oxidative cascade markers.

2.5. Determination of Hepatic-Specific Markers. Serum levels of aspartate aminotransferase (AST) and alanine aminotransferase (ALT) were assessed according to Reitman and Frankel [26], while that of alkaline phosphatase (ALP) was determined according to Tietz et al. [27]. Furthermore, gamma-glutamyl transferase $(\gamma \mathrm{GT})$ and lactate dehydrogenase $(\mathrm{LDH})$ activities were evaluated according to Szasz [28] and Babson and Babson [29], respectively.

2.6. Determination of Proinflammatory Cytokines. IL-1 $\beta$, IL6 , and TNF- $\alpha$ proinflammatory markers were assayed using commercial ELISA kits according to the manufacturer's instructions, and the absorbance values were measured using an automated ELISA reader at $450 \mathrm{~nm}$.

2.7. Evaluation of Liver and Brain Oxidative/Antioxidative Markers. The oxidant/antioxidant status was evaluated in the previously prepared liver and brain tissue homogenates through determination of malondialdehyde (MDA) [30] and nitric oxide (NO) [31] concentrations. In addition, the 
content of antioxidant molecules including reduced glutathione (GSH), superoxide dismutase (SOD), catalase (CAT), and glutathione peroxidase (GSH-Px) was also determined in the liver and brain according to Beutler et al. [32], Nishikimi et al. [33], Aebi [34], and Paglia and Valentine [35], respectively.

2.8. Statistical Analysis. All results were presented as the mean \pm SE from eight mice per group. The results were analyzed statistically by using a statistical program (version 21.0; SPSS Inc., Chicago, IL, USA). First, all data were tested for normality (Shapiro-Wilk's test) and homogeneity (Levene's test) as well. Then, one-way analysis of variance (ANOVA) was used to analyze the relationship between multiple groups, followed by Tukey's test under a probability of 0.05 .

\section{Results}

3.1. Effect of TQ and/or PP Treatment on Hepatic Markers. As depicted in Figure 1, MC caused hepatotoxicity indicated by significant $(P<0.05)$ elevations of serum AST, ALT, ALP, $\gamma \mathrm{GT}$, and LDH levels of 234.34\%, 229.74\%, 214.33\%, $214.15 \%$, and $162.17 \%$, respectively, when compared to the normal controls. On the other hand, the treatment of MCintoxicated mice with TQ or PP exerted significant improvements in these hepatic markers denoted by marked $(P<0.05)$ decreases in AST, ALT, ALP, $\gamma$ GT, and LDH levels (MC-TQ: $73.11 \%, 76.42 \%, 65.37 \%, 63.03 \%$, and $71.72 \%$ and MC-PP: $82.40 \%, 94.85 \%, 78.54 \%, 68.49 \%$, and $82.33 \%$, respectively) in comparison to MC-treated animals (Figure 1). Obviously, when TQ and PP were given in combination, they could significantly $(P<0.05)$ restore all previous parameters close to the normal values than their individual treatments to reach $48.01 \%, 52.56 \%, 48.56 \%, 48.31 \%$, and $65.74 \%$, respectively, when compared to the $\mathrm{MC}$ values.

3.2. Effect of the TQ and/or PP Treatment on Inflammatory Reaction. Serum concentrations of inflammatory markers (IL-1 $\beta$, IL-6, and TNF- $\alpha$ ) were dramatically $(P<0.05)$ higher in the MC-treated mice than in the saline-treated ones $(323.99 \%, 289.12 \%$, and $323.00 \%$, respectively) as shown in Figure 2. However, treatment of MC-intoxicated mice with TQ or PP significantly $(P<0.05)$ lowered serum IL- $1 \beta$, IL6 , and TNF- $\alpha$ concentrations (MC-TQ: $40.52 \%, 43.85 \%$, and $38.68 \%$ and MC-PP: $48.85 \%, 53.95 \%$, and $50.97 \%$, respectively) in comparison to the MC group. Interestingly, the MC-TQ-PP group showed marked decreases in the measured inflammatory cytokines to $33.29 \%, 37.26 \%$, and $34.37 \%$, respectively, relative to $\mathrm{MC}$ alone suggesting that the anti-inflammatory effects of the TQ-and-PP-combined therapy were more effective than those of each individual treatment (Figure 2).

3.3. Effect of the $T Q$ and/or $P P$ Treatment on Hepatic Oxidant/Antioxidant Status. As shown in Figure 3, MC evoked oxidative damage in the liver that was evident by dramatic $(P<0.05)$ increases in MDA and NO concentrations $(252.83 \%$ and $236.52 \%$, respectively) and decreases in GSH, SOD, CAT, and GSH-Px contents (39.42\%, 28.17\%, 28.42\%, and $28.85 \%$, respectively) in comparison to the corresponding control group. Alternatively, treatment with TQ, PP, or both attenuated the MC-induced hepatooxidative damage indicated by marked $(P<0.05)$ reductions in MDA $(63.90 \%$, $70.48 \%$, and $41.37 \%$, respectively) and NO $(67.26 \%, 74.10 \%$, $45.12 \%$, respectively) when compared with MC-intoxicated animals. Further, it was observed that each of the TQ and PP was able to significantly $(P<0.05)$ elevate the MCreduced GSH levels and SOD, CAT, and GSH-Px activities (MC-TQ: $211.18 \%, 243.57 \%, 271.96 \%$, and $266.34 \%$ and MC-PP: $189.57 \%, 207.60 \%, 230.14 \%$, and $226.48 \%$, respectively) as compared with the MC values. In addition, the cotreatment of TQ and PP boosted the antioxidant molecules more profoundly than each single regimen seen by marked $(P<0.05)$ increases in the hepatic GSH, SOD, CAT, and GSH-Px contents by $242.09 \%, 316.91 \%, 324.46 \%$, and $321.52 \%$, respectively, relative to that of the MC group.

3.4. Effect of the TQ and/or PP Treatment on Brain Oxidant/Antioxidant Status. Figure 4 displays that MC provoked drastic $(P<0.05)$ increases in brain MDA and NO concentrations (245.15\% and $190.04 \%$, respectively) as compared with the control group. Our results also revealed a reduced antioxidant capacity depicted by marked $(P<0.05)$ decreases in the GSH level and SOD, CAT, and GSH-Px activities (43.85\%, 51.33\%, 52.76\%, and $41.97 \%$, respectively) in the brain tissue of MC-intoxicated mice. Nevertheless, treatment with TQ or PP was effective in diminishing oxidative damage induced by MC, wherein TQ could obviously $(P<0.05)$ lower the MDA and NO levels (62.77\% and $75.11 \%$, respectively) accompanied by significant $(P<0.05)$ increases in brain GSH, SOD, CAT, and GSH-Px contents (169.90\%, 159.73\%, $157.73 \%, 165.70 \%$, respectively) in comparison to toxic controls. In the same line, PP could markedly $(P<0.05)$ decrease the levels of the MC-increased MDA and NO to $67.72 \%$ and $82.44 \%$, respectively, and elevate the contents of GSH, SOD, CAT, and GSH-Px in brain tissue (152.02\%, $145.80 \%$, $148.02 \%$, and $138.69 \%$, respectively) relative to MC controls. Notably, combined therapy showed better improvement in the antioxidant status of brain tissue of MC-treated mice proven by significant $(P<0.05)$ reductions in MDA and NO levels (43.81\% and 57.15\%, respectively) and upregulated GSH level and SOD, CAT and GSH-Px activities (223.82\%, $183.77 \%, 177.62 \%$, and $231.15 \%$, respectively) in comparison with the initial MC values (Figure 4).

\section{Discussion}

Extensive anthropogenic eutrophication of water bodies leads to growth of cyanobacteria and consequently the production of high amounts of microcystin- (MC-) LR which is a potent environmental hazard to human, animal, fish, and bird health. Consumption of contaminated food and drinking water is the main source of exposure [1]. It is well known that the liver is the primary target of MC intoxication $[4,15]$. In the same line, our study revealed severe hepatotoxicity after MC treatment indicated by marked elevations of serum AST, ALT, and ALP levels. These findings support the data obtained by previous reports $[1,36$, 37] which studied the change in liver function during $M C$ 


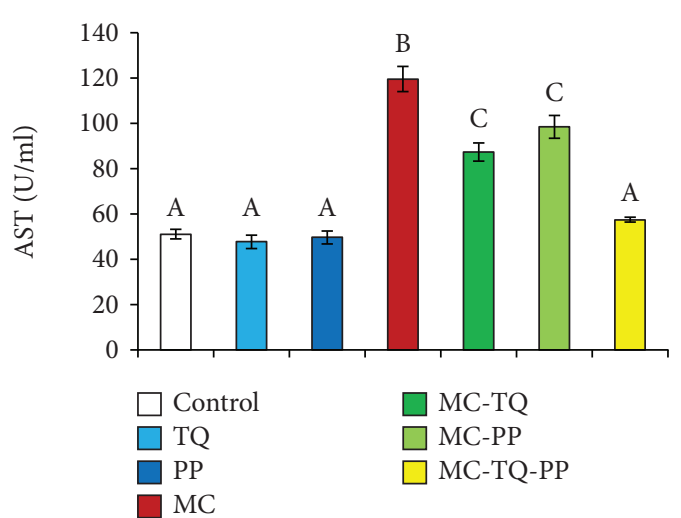

(a)

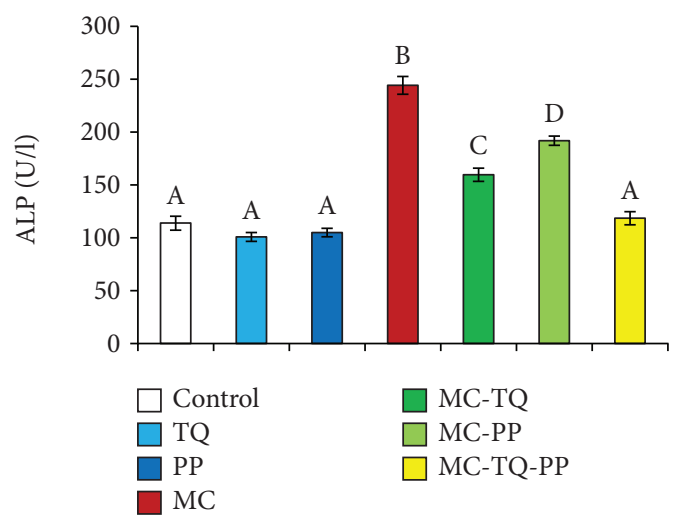

(c)

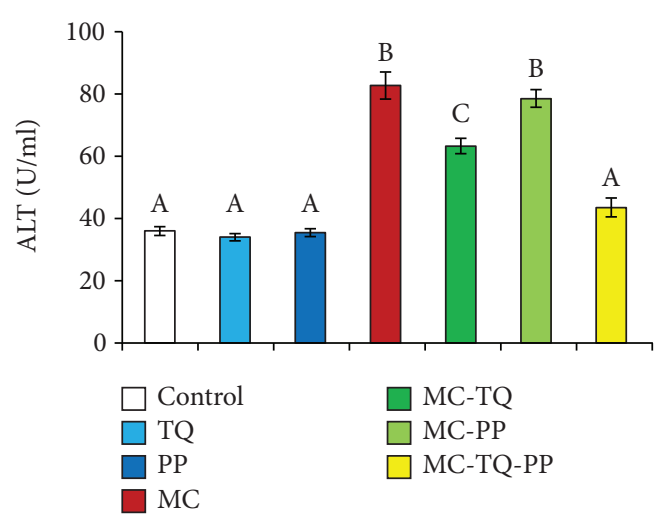

(b)

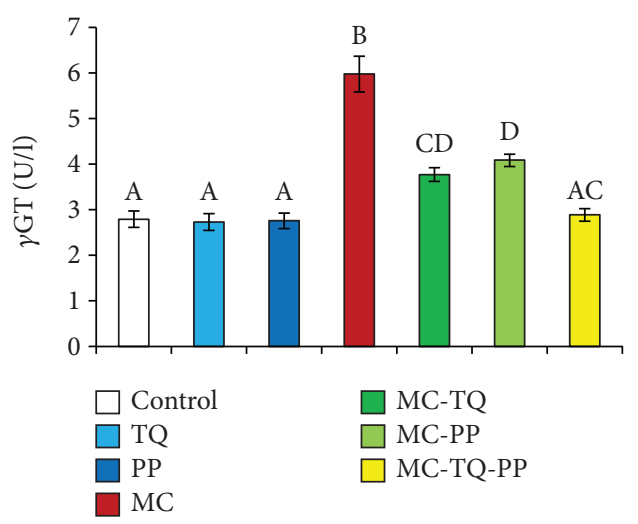

(d)

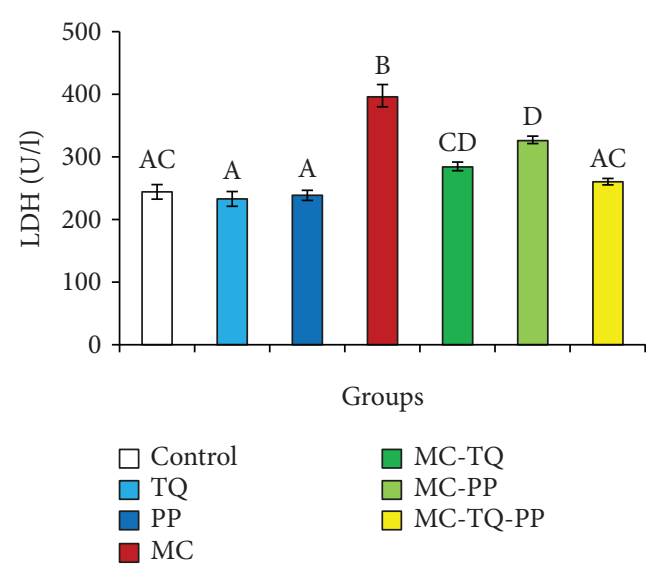

(e)

FIGURE 1: Ameliorative effect of thymoquinone (TQ) and/or piperine (PP) against microcystin- (MC-) LR-induced hepatotoxicity in mice. (a) AST; (b) ALT; (c) ALP; (d) $\gamma$ GT; (e) LDH. Values are expressed as mean \pm SE. Columns carrying different letters are considered significantly different $(P<0.05)$.

insult. Previous studies have attributed the MC-induced hepatotoxicity to the high affinity of MC to form strong covalent bonds with hepatic serine/threonine-specific protein phosphatases which potently inhibit their activities [14]. These events initiate a cascade of cellular dysfunction including disturbing cellular signaling and loss of cytoskeletal integrity due to protein hyperphosphorylation, ending in the leakage of liver marker enzymes into the bloodstream $[4,5,15,38]$.

Moreover, a growing body of evidence suggests the involvement of oxidative stress in MC-induced hepatotoxicity
$[1,6,14,37,39]$. Besides the hepatotoxicity, $\mathrm{MC}$ has the ability to cross the blood-brain barrier [40] and cause neurotoxicity via possible mechanisms of phosphatase inhibition and oxidative damage $[2,5,41]$. In the biological system, oxidative stress comprises a sequence of events which involves the excessive production of $\mathrm{ROS}\left(\mathrm{O}_{2}{ }^{--}\right.$(superoxide anions), $\mathrm{OH}^{\bullet}$ (hydroxyl radicals), $\mathrm{H}_{2} \mathrm{O}_{2}$ (hydrogen peroxide), and $\mathrm{NO}$ (nitric oxide)) that overwhelms the cellular antioxidant defense mechanisms. These events cause several deleterious effects on cellular macromolecules such as lipid peroxidation, mitochondrial 


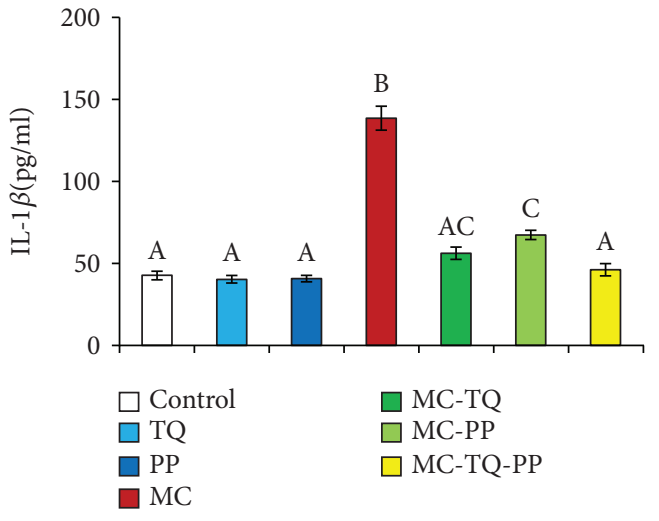

(a)

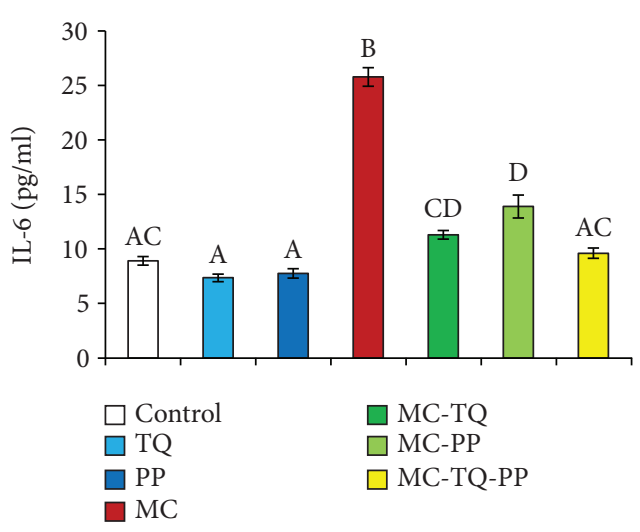

(b)

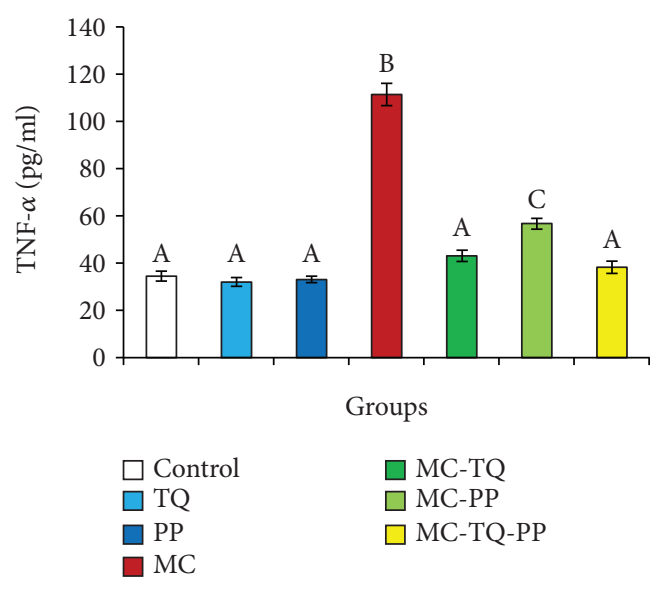

(c)

FIGURE 2: Ameliorative effect of thymoquinone (TQ) and/or piperine (PP) against microcystin- (MC-) LR-induced inflammation in mice. (a) IL-1 $\beta$; (b) IL-6; (c) TNF- $\alpha$. Values are expressed as mean \pm SE. Columns carrying different letters are considered significantly different $(P<0.05)$.

dysfunction, ATP reduction, protein oxidation, and DNA damage $[38,42,43] . \mathrm{OH}^{\bullet}$ is the most harmful ROS that directly attacks the lipid content of the cellular membranes causing lipid peroxidation manifested by increased production of MDA that also has the affinity to bind to other cell molecules which worsens the situation. Among cellular antioxidants, GSH, CAT, and GSH-Px are required for hydrolysis of $\mathrm{H}_{2} \mathrm{O}_{2}$ into $\mathrm{H}_{2} \mathrm{O}$. However, depletion of such antioxidants makes the hydrolysis reaction shift to Fenton's reaction, where large amounts of $\mathrm{OH}^{\bullet}$ are formed from $\mathrm{H}_{2} \mathrm{O}_{2}$ [43]. Consistently, this study suggests the implication of oxidative stress during $\mathrm{MC}$ intoxication which was observed by significant increases in MDA and NO levels along with the depletion of cellular antioxidants in liver and brain tissues. In addition, in the current study, loss of hepatocyte membrane integrity due to MC-evoked lipid peroxidation may be another cause of the efflux of transaminases into the bloodstream $[38,42]$.

Furthermore, MC has been reported to accumulate in the liver as a part of its detoxification process, wherein it forms a conjugate with hepatic GSH, namely, the MC-GSH complex [14]. That leads to exhaustion of the available GSH which is required for the recycling of GSH-Px during the hydrolysis reaction of $\mathrm{H}_{2} \mathrm{O}_{2}$ into $\mathrm{H}_{2} \mathrm{O}$ [44]. Our data also showed decreases in SOD activities which are the main $\mathrm{O}_{2}{ }^{--}$scavenger. Therefore, the accumulated $\mathrm{O}_{2}{ }^{\bullet-}$ spontaneously reacts with the excess $\mathrm{NO}$ and forms the peroxynitrite radical $\left(\mathrm{ONOO}^{-}\right)$ which causes protein nitration and dysfunction and depletion of GSH $[25,44]$, recently called nitrosative stress.

$\gamma \mathrm{GT}$ is described as a sensitive marker for oxidative stress [45]. $\gamma \mathrm{GT}$ is a membranous protein expressed in many cell types, particularly in the liver. It is expressed mainly in the apical plasma membrane facing the extracellular space [46], where it functions as a GSH recycler [47]. Therefore, we suppose that the increased serum level of $\gamma \mathrm{GT}$ and decreased tissue concentrations of GSH may be attributed to the loss of membrane integrity and release of $\gamma \mathrm{GT}$ into the bloodstream as a result of $\mathrm{MC}$-induced lipid peroxidation along with reduced GSH recycling. Our data confirm the studies which reported an inverse correlation between $\gamma \mathrm{GT}$ and antioxidants [45]. The current study also reported a significant increase in the serum LDH level. Since the cell integrity is lost due to lipid peroxidation, $\mathrm{LDH}$ is released extracellularly and increases its serum level [48]. It is well known that when there is a reduction in ATP production along the electron transport chain during the aerobic glycolysis, the reaction shifts to anaerobic glycolysis where lactates and LDH are increased $[25,48]$. Our group has previously demonstrated 


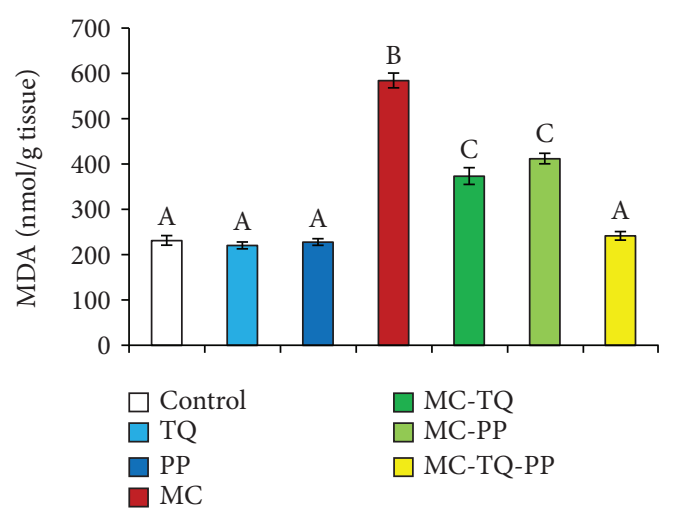

(a)

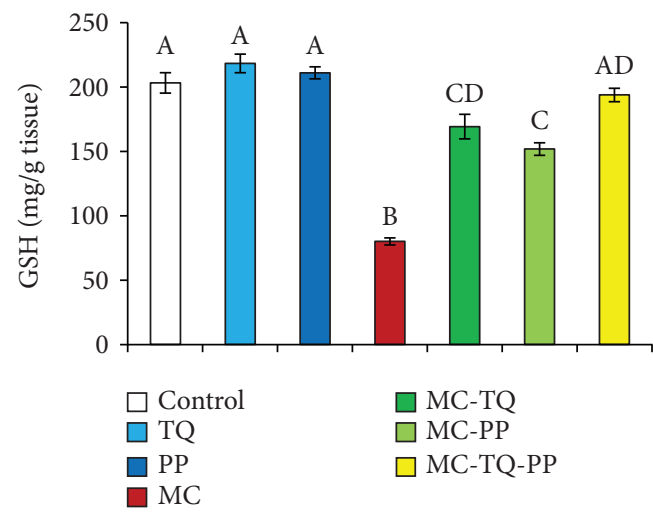

(c)

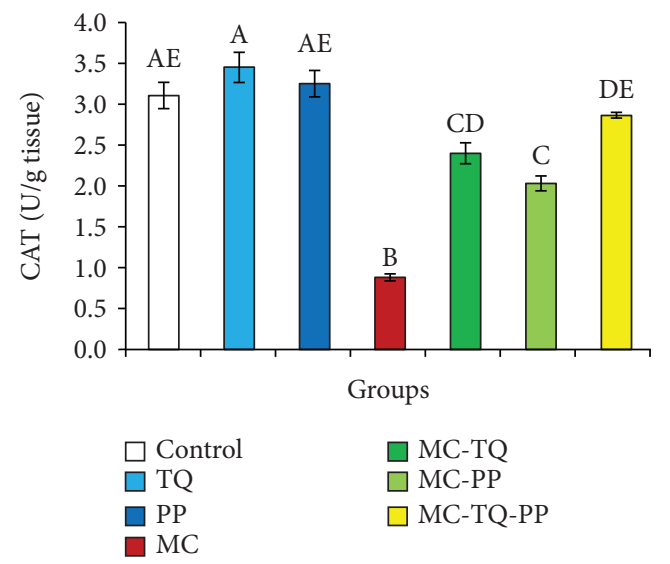

(e)

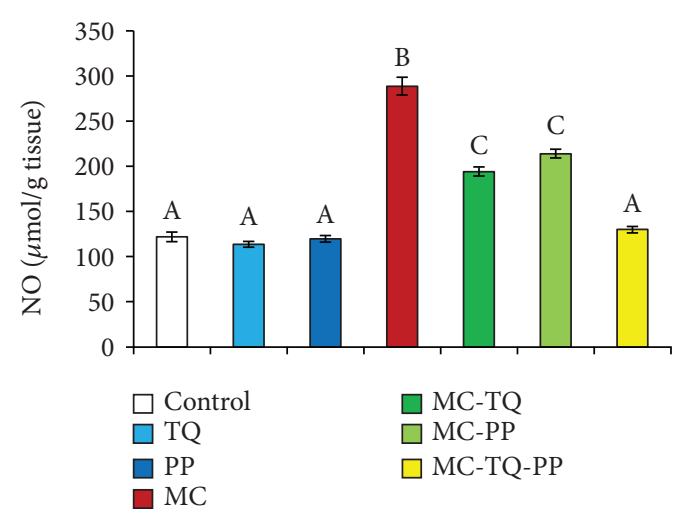

(b)

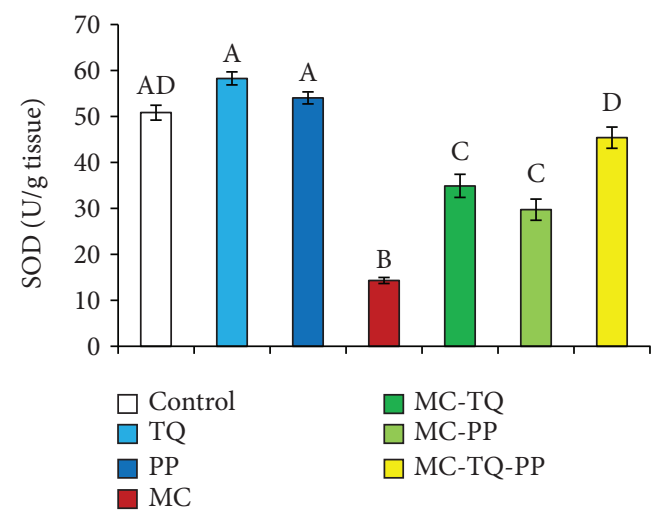

(d)

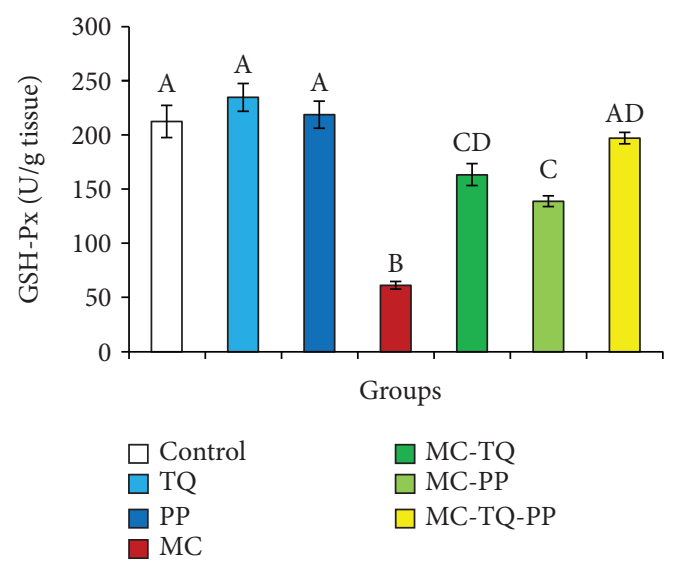

(f)

FIGURE 3: Ameliorative effect of thymoquinone (TQ) and/or piperine (PP) against microcystin- (MC-) LR-induced oxidative damage in the mouse liver. (a) MDA; (b) NO; (c) GSH; (d) SOD; (e) CAT; (f) GSH-Px. Values are expressed as mean \pm SE. Columns carrying different letters are considered significantly different $(P<0.05)$.

an increased serum LDH in a fipronil-targeted electron transport chain [38]. Therefore, we suppose that the electron transport chain might be a possible target for MC.

The present study reported marked increases in serum $\gamma \mathrm{GT}$ and tissue NO, besides the intracellular accumulation of MC. All of these events played a central role in GSH exhaustion and subsequent reduction in GSH-Px, SOD, and CAT activities. Taken altogether, our data confirm the involvement of oxidative damage in MC-induced toxicity $[1,2,6,14,37,39,41]$.
It was well known that excessive ROS production and oxidative stress initiate an intracellular cascade signaling that enhances the expression of the proinflammatory gene as well as the production of inflammatory cytokines [49]. Along with these, the present investigation revealed increases in serum IL- $1 \beta$, IL-6, and TNF- $\alpha$ which might happen in response to $\mathrm{MC}$-induced oxidative stress in the liver and brain tissue. The ongoing findings are in agreement with Ahmad et al. [1], Abd AL Majeed et al. [50], and Lone et al. [25]. The current data also emphasize our previous 


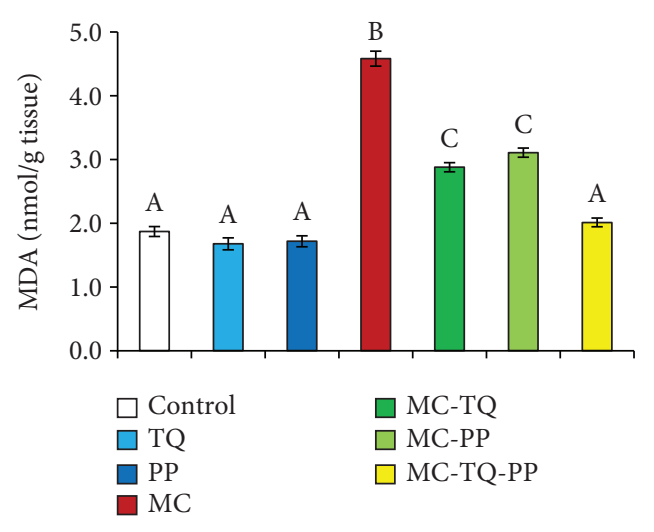

(a)

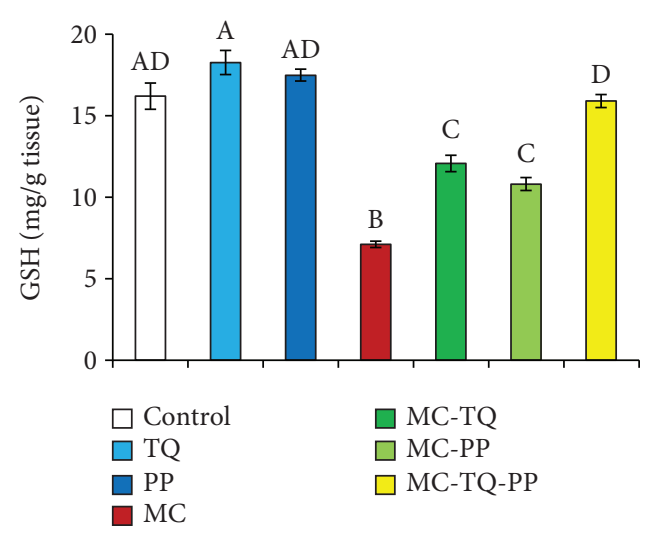

(c)

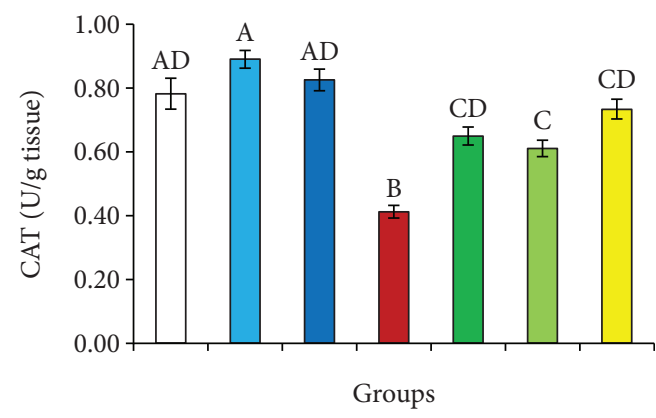

(e)

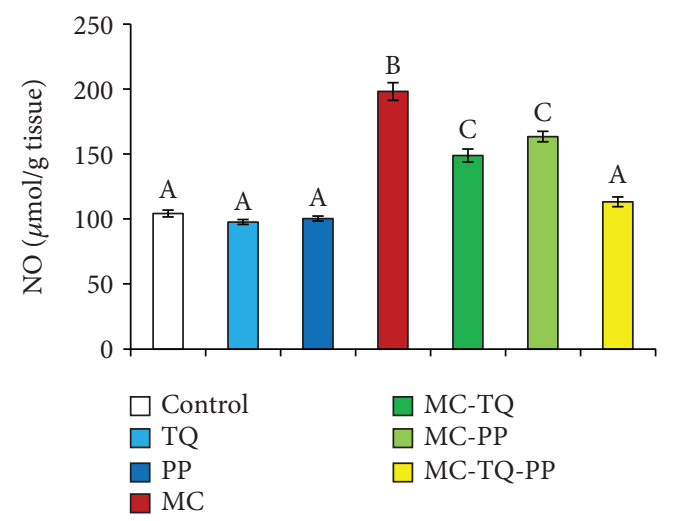

(b)

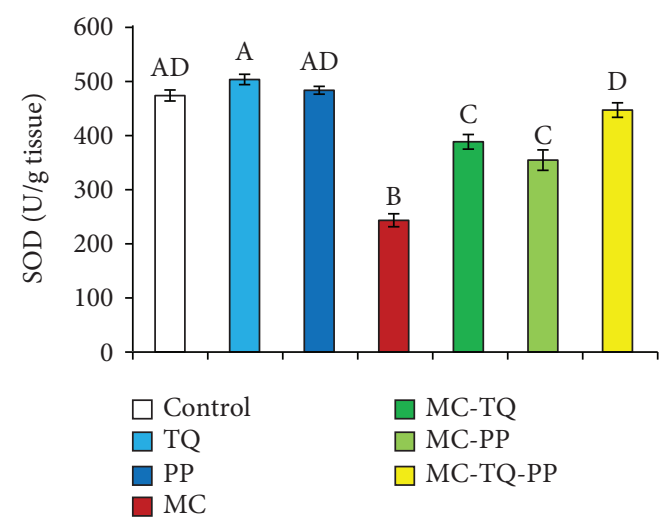

(d)

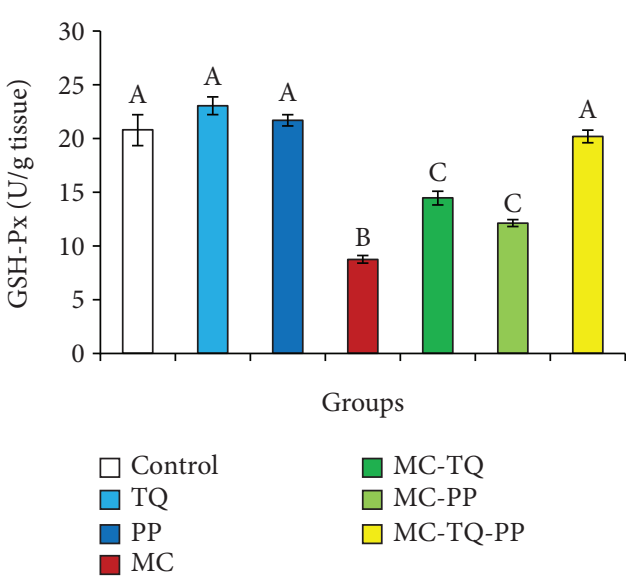

(f)

FIGURE 4: Ameliorative effect of thymoquinone (TQ) and/or piperine (PP) against microcystin- (MC-) LR-induced oxidative damage in the mouse brain. (a) MDA; (b) NO; (c) GSH; (d) SOD; (e) CAT; (f) GSH-Px. Values are expressed as mean \pm SE. Columns carrying different letters are considered significantly different $(P<0.05)$.

reports which demonstrated a triggered inflammatory reaction in response to oxidative damage induced by piroxicam [51], acetaminophen [52], fipronil insecticide [38], and cadmium [42] insults. Basically, the oxidative insult besides the proinflammatory cytokine, recorded in the present result, increases the vulnerability of the brain to various neurodegenerative diseases.
TQ is described to have anti-inflammatory and antioxidant properties exerted by its ROS scavenging and antioxidant upregulation activities $[17,18]$. PP is also reported to have the same pharmacological actions [20,21]. In the present investigation, treatment with TQ or PP attenuated the MC-induced hepatotoxicity and neurotoxicity indicated by significant reduction of the serum biochemical parameters 


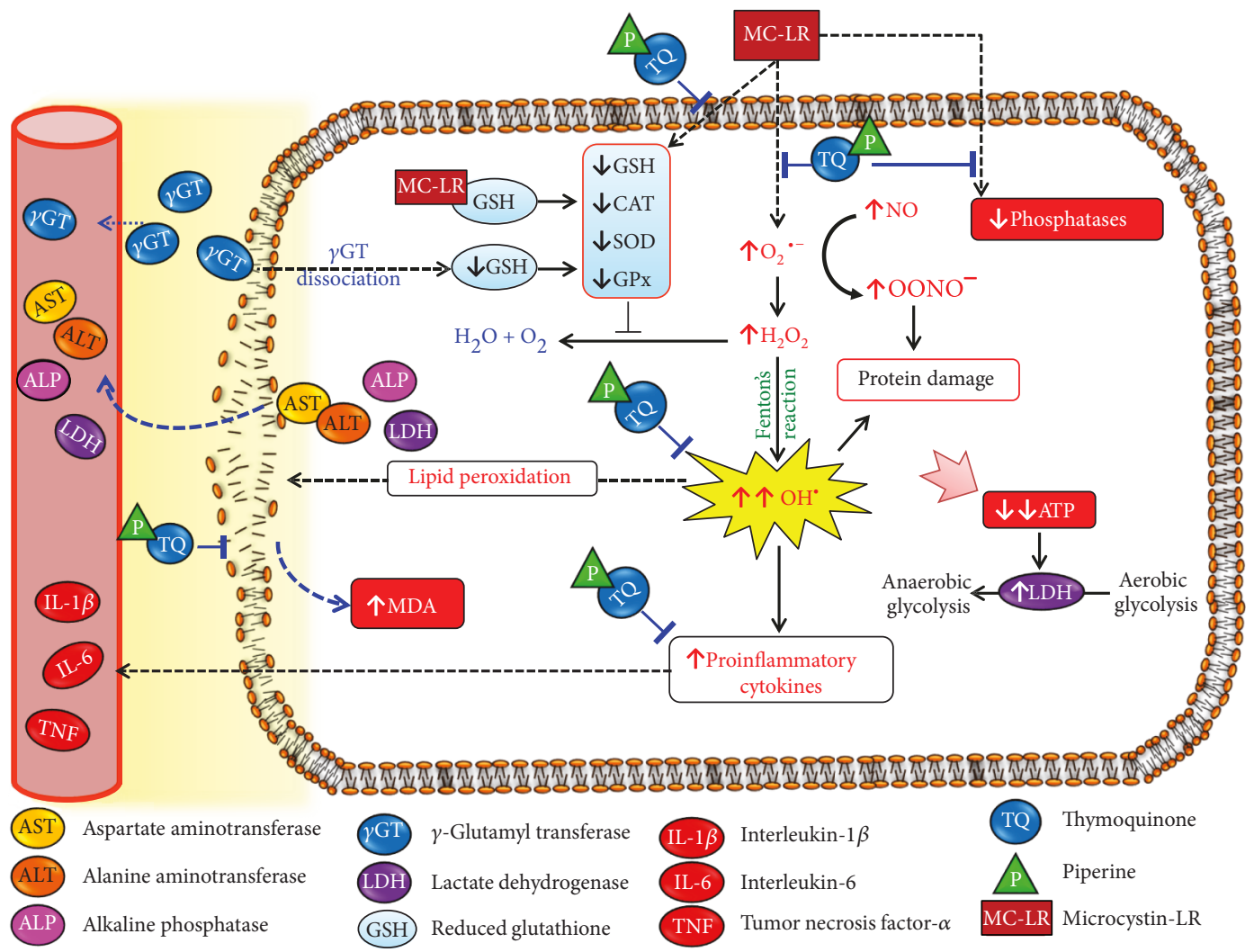

FIGURE 5: Schematic diagram of the possible antioxidant and anti-inflammatory effects of thymoquinone (TQ), piperine (PP), and their combination against microcystin- (MC-) LR-induced hepatotoxicity and neurotoxicity in mice.

and proinflammatory cytokines along with improvement in the oxidative/antioxidative state. In fact, the chemical structure of TQ and PP is the key of their antioxidant activity. Their chemical structures contain a phenol ring, double bonds, and hydrogen atom $\left(\mathrm{H}^{+}\right)$allowing free delocalization of electrons and $\mathrm{H}^{+}$donation by which scavenging and neutralization of ROS, NO, and accumulated MC take place and eventually attenuate the oxidative damage [52]. Supplementation with external ROS scavenging agents increases the availability of the cellular unconjugated GSH promoting the cellular antioxidant capacity. In another cohort study, dietary supplementation of antioxidants has shown a negative correlation with serum $\gamma \mathrm{GT}$ suggesting the improvement of cellular oxidative status [45].

Interestingly, cotherapy of TQ and PP offers plenty of phenol rings and $\mathrm{H}^{+}$donors by which they could obviously improve all parameters much better than their individual treatments. PP is described as a dietary enhancer used to increase the bioavailability of other antioxidants either by promoting their absorption or by slowing their biodegradation in the liver via inhibition of several cytochrome P450mediated metabolic pathways [53]. PP has been reported to enhance the bioavailability of ginsenoside Rh2 [54], puerarin [22], and (-)-epigallocatechin-3-gallate [23]. Talib (2017) has documented a synergistic action between TQ and PP against breast cancer in a mouse model. Therefore, we assume that the enhanced protective effects of the combined treatment were likely due to the synergistic antioxidant capacity of both agents and the increased bioavailability of TQ. Figure 5 shows the possible antioxidant and anti-inflammatory effects of TQ, PP, and their combination against MC-induced hepatotoxicity and neurotoxicity.

\section{Conclusion}

In the current study, MC provokes hepatotoxic and neurotoxic effects indicated by elevated serum hepatic markers and proinflammatory cytokines along with disruption of the oxidative state. An electron transport chain may be a possible target for MC. Treatment with TQ or PP alone alleviates such injuries in MC-intoxicated animals which may be attributed to their antioxidant and anti-inflammatory properties. Notably, a combination of TQ and PP exerts more improvement than their individual treatments. Such efficacy might be due to providing more than one antioxidant and due to the PP-enhanced bioavailability of TQ.
Abbreviations
ALT: Alanine aminotransferase
ALP: $\quad$ Alkaline phosphatase
AST: Aspartate aminotransferase
CAT: Catalase
$\gamma \mathrm{GT}$ : $\quad$ Gamma-glutamyl transferase
GSH: Reduced glutathione
GSH-Px: Glutathione peroxidase
IL-1 $\beta$ : Interleukin- $1 \beta$
IL-6: $\quad$ Interleukin-6 


\author{
LDH: $\quad$ Lactate dehydrogenase \\ MDA: Malondialdehyde \\ SOD: $\quad$ Superoxide dismutase \\ TNF- $\alpha$ : Tumor necrosis factor- $\alpha$ \\ TQ: Thymoquinone \\ MC-LR: Microcystin-LR.
}

\section{Data Availability}

The data used to support the findings of this study are available from the corresponding author upon request.

\section{Conflicts of Interest}

The authors declare that there are no conflicts of interest.

\section{Acknowledgments}

The authors extend their appreciation to the Deanship of Scientific Research at King Saud University for funding this work through research group number RGP-018. They also are grateful to the Department of Veterinary Forensic Medicine and Toxicology, Benha University, for the technical support provided.

\section{References}

[1] W. Ahmad, E. Haque, F. Jamshed, A. Saad, and S. Al-Jasabi, "Prevention of formation of toxic carbonyl and aldehyde compounds in BALB/C micehepatocytes caused by the toxicity of microcystin-LR using hydroxytyrosol extracted from Zizyphusspina-christi," American-Eurasian Journal of Toxicological Sciences, vol. 6, pp. 68-73, 2014.

[2] Y. Hu, L. Chen, J. Chen, X. Zhang, and P. Xie, "A review of reproductive toxicity of microcystins," Journal of Hazardous Materials, vol. 301, pp. 381-399, 2016.

[3] WHO, "Cyanobacterial toxins: microcystin-LR in drinkingwater," Environmental Health, vol. 2, pp. 1-14, 1998.

[4] A. Campos and V. Vasconcelos, "Molecular mechanisms of microcystin toxicity in animal cells," International Journal of Molecular Sciences, vol. 11, no. 1, pp. 268-287, 2010.

[5] O. Myhre, D. M. Eide, S. Kleiven, H. C. Utkilen, and T. Hofer, "Repeated five-day administration of L-BMAA, microcystinLR, or as mixture, in adult C57BL/6 mice - lack of adverse cognitive effects," Scientific Reports, vol. 8, no. 1, pp. 2308-2314, 2018.

[6] J. Jiang, Z. Shan, W. Xu et al., "Microcystin-LR induced reactive oxygen species mediate cytoskeletal disruption and apoptosis of hepatocytes in Cyprinus carpio L," PLoS One, vol. 8, no. 12, article e84768, 2013.

[7] J. Chen and P. Xie, "Seasonal dynamics of the hepatotoxic microcystins in various organs of four freshwater bivalves from the large eutrophic Lake Taihu of subtropical China and the risk to human consumption," Environmental Toxicology, vol. 20, no. 6, pp. 572-584, 2005.

[8] Z. A. Mohamed, W. W. Carmichael, and A. A. Hussein, "Estimation of microcystins in the freshwater fish Oreochromis niloticus in an Egyptian fish farm containing a microcystis bloom," Environmental Toxicology, vol. 18, no. 2, pp. 137141, 2003.
[9] A. Yokoyama and H. D. Park, "Mechanism and prediction for contamination of freshwater bivalves (Unionidae) with the cyanobacterial toxin microcystin in hypereutrophic Lake Suwa, Japan," Environmental Toxicology, vol. 17, no. 5, pp. 424-433, 2002.

[10] Z. Romanowska-Duda, J. Mankiewicz, M. Tarczynska, Z. Walter, and M. Zalewski, "The effect of toxic cyanobacteria (blue-green algae) on water plants and animal cells," Polish Journal of Environmental Studies, vol. 11, pp. 561566, 2002.

[11] E. M. Jochimsen, W. W. Carmichael, J. S. An et al., "Liver failure and death after exposure to microcystins at a hemodialysis center in Brazil," The New England Journal of Medicine, vol. 338, no. 13, pp. 873-878, 1998.

[12] S. M. F. O. Azevedo, W. W. Carmichael, E. M. Jochimsen et al., "Human intoxication by microcystins during renal dialysis treatment in Caruaru-Brazil," Toxicology, vol. 181-182, pp. 441-446, 2002.

[13] P. Xu, X. X. Zhang, C. Miao et al., "Promotion of melanoma cell invasion and tumor metastasis by microcystin-LR via phosphatidylinositol 3-kinase/AKT pathway," Environmental Science \& Technology, vol. 47, pp. 8801-8808, 2013.

[14] B. Greer, J. P. Meneely, and C. T. Elliott, "Uptake and accumulation of microcystin-LR based on exposure through drinking water: an animal model assessing the human health risk," Scientific Reports, vol. 8, no. 1, article 4913, 2018.

[15] N. A. Robinson, C. F. Matson, and J. G. Pace, “Association of microcystin-LR and its biotransformation product with a hepatic-cytosolic protein," Journal of Biochemical Toxicology, vol. 6, no. 3, pp. 171-180, 1991.

[16] K. Wang, S. Garg, and T. D. Waite, "Light-mediated reactive oxygen species generation and iron redox transformations in the presence of exudate from the cyanobacterium microcystis aeruginosa," Environmental Science \& Technology, vol. 51, no. 15 , pp. 8384-8395, 2017.

[17] I. Ö. Aycan, A. Tüfek, O. Tokgöz et al., "Thymoquinone treatment against acetaminophen-induced hepatotoxicity in rats," International Journal of Surgery, vol. 12, no. 3, pp. 213-218, 2014.

[18] A. Mabrouk, I. Bel Hadj Salah, W. Chaieb, and H. Ben Cheikh, "Protective effect of thymoquinone against lead-induced hepatic toxicity in rats," Environmental Science and Pollution Research, vol. 23, no. 12, pp. 12206-12215, 2016.

[19] Y. S. Abulfadl, N. N. El-Maraghy, A. A. E. Ahmed, S. Nofal, Y. Abdel-Mottaleb, and O. A. Badary, "Thymoquinone alleviates the experimentally induced Alzheimer's disease inflammation by modulation of TLRs signaling," Human \& Experimental Toxicology, vol. 37, no. 10, pp. 1092-1104, 2018.

[20] K. Srinivasan, "Black pepper and its pungent principle-piperine: a review of diverse physiological effects," Critical Reviews in Food Science and Nutrition, vol. 47, no. 8, pp. 735-748, 2007.

[21] R. S. Vijayakumar and N. Nalini, "Efficacy of piperine, an alkaloidal constituent from Piper nigrum on erythrocyte antioxidant status in high fat diet and antithyroid drug induced hyperlipidemic rats," Cell Biochemistry and Function, vol. 24, no. 6, pp. 491-498, 2006.

[22] Y. Z. Liang, H. M. Chen, Z. Q. Su et al., "White pepper and piperine have different effects on pharmacokinetics of puerarin in rats," Evidence-Based Complementary and Alternative Medicine, vol. 2014, Article ID 796890, 8 pages, 2014.

[23] J. D. Lambert, J. Hong, D. H. Kim, V. M. Mishin, and C. S. Yang, "Piperine enhances the bioavailability of the tea 
polyphenol (-)-epigallocatechin-3-gallate in mice," The Journal of Nutrition, vol. 134, no. 8, pp. 1948-1952, 2004.

[24] K. M. Alkharfy, A. Ahmad, R. M. A. Khan, and W. M. AlShagha, "Pharmacokinetic plasma behaviors of intravenous and oral bioavailability of thymoquinone in a rabbit model," European Journal of Drug Metabolism and Pharmacokinetics, vol. 40, no. 3, pp. 319-323, 2015.

[25] Y. Lone, M. Bhide, and R. K. Koiri, "Amelioratory effect of coenzyme Q10 on potential human carcinogen microcystinLR induced toxicity in mice," Food and Chemical Toxicology, vol. 102, pp. 176-185, 2017.

[26] S. Reitman and S. Frankel, "A colorimetric method for the determination of serum glutamic oxalacetic and glutamic pyruvic transaminases," American Journal of Clinical Pathology, vol. 28, no. 1, pp. 56-63, 1957.

[27] N. W. Tietz, C. A. Burtis, P. Duncan et al., "A reference method for measurement of alkaline phosphatase activity in human serum," Clinical Chemistry, vol. 29, no. 5, pp. 751761, 1983.

[28] G. Szasz, "Reaction-rate method for $\gamma$-glutamyltransferase activity in serum," Clinical chemistry, vol. 22, pp. 2051-2055, 1976.

[29] A. L. Babson and S. R. Babson, "Kinetic colorimetric measurement of serum lactate dehydrogenase activity," Clinical Chemistry, vol. 19, no. 7, pp. 766-769, 1973.

[30] M. Mihara and M. Uchiyama, "Determination of malonaldehyde precursor in tissues by thiobarbituric acid test," Analytical Biochemistry, vol. 86, no. 1, pp. 271-278, 1978.

[31] L. C. Green, D. A. Wagner, J. Glogowski, P. L. Skipper, J. S. Wishnok, and S. R. Tannenbaum, "Analysis of nitrate, nitrite, and [15N]nitrate in biological fluids," Analytical Biochemistry, vol. 126, no. 1, pp. 131-138, 1982.

[32] E. Beutler, O. Duron, and B. M. Kelly, "Improved method for determination of blood glutathione," The Journal of Laboratory and Clinical Medicine, vol. 61, pp. 882-888, 1963.

[33] M. Nishikimi, N. A. Rao, and K. Yagi, "The occurrence of superoxide anion in the reaction of reduced phenazine methosulfate and molecular oxygen," Biochemical and Biophysical Research Communications, vol. 46, no. 2, pp. 849854, 1972.

[34] H. Aebi, “Catalase in vitro," Methods in Enzymology, vol. 105, pp. 121-126, 1984.

[35] D. E. Paglia and W. N. Valentine, "Studies on the quantitative and qualitative characterization of erythrocyte glutathione peroxidase," The Journal of Laboratory and Clinical Medicine, vol. 70, no. 1, pp. 158-169, 1967.

[36] J. Chen, P. Xie, L. Li, and J. Xu, "First identification of the hepatotoxic microcystins in the serum of a chronically exposed human population together with indication of hepatocellular damage," Toxicological Sciences, vol. 108, no. 1, pp. 81-89, 2009.

[37] J. Chen, Z. Liu, G. Zhou, Z. Han, H. Zhang, and Y. Zhang, "Effect of cyanobacteria extract on some associated enzymes in mouse liver in vivo," Chinese Journal of Oceanology and Limnology, vol. 28, no. 6, pp. 1180-1186, 2010.

[38] M. M. Abdel-Daim and A. Abdeen, "Protective effects of rosuvastatin and vitamin $\mathrm{E}$ against fipronil-mediated oxidative damage and apoptosis in rat liver and kidney," Food and Chemical Toxicology, vol. 114, pp. 69-77, 2018.

[39] W. X. Ding, H. M. Shen, H. G. Zhu, and C. N. Ong, "Studies on oxidative damage induced by cyanobacteria extract in primary cultured rat hepatocytes," Environmental Research, vol. 78, no. 1, pp. 12-18, 1998.

[40] W. J. Fischer, S. Altheimer, V. Cattori, P. J. Meier, D. R. Dietrich, and B. Hagenbuch, "Organic anion transporting polypeptides expressed in liver and brain mediate uptake of microcystin," Toxicology and Applied Pharmacology, vol. 203, no. 3, pp. 257-263, 2005.

[41] M. Maidana, V. Carlis, F. G. Galhardi et al., "Effects of microcystins over short- and long-term memory and oxidative stress generation in hippocampus of rats," Chemico-Biological Interactions, vol. 159, no. 3, pp. 223-234, 2006.

[42] A. Abdeen, A. Ghonim, R. El-Shawarby et al., "Protective effect of cinnamon against cadmium-induced hepatorenal oxidative damage in rats," International Journal of Pharmacology and Toxicology, vol. 5, no. 1, p. 17, 2017.

[43] H. Sies, C. Berndt, and D. P. Jones, "Oxidative stress," Annual Review of Biochemistry, vol. 86, no. 1, pp. 715-748, 2017.

[44] J. Nordberg and E. S. J. Arnér, "Reactive oxygen species, antioxidants, and the mammalian thioredoxin system," Free Radical Biology and Medicine, vol. 31, no. 11, pp. 1287-1312, 2001.

[45] D. H. Lee, R. Blomhoff, and D. R. Jacobs, "Review: is serum gamma glutamyltransferase a marker of oxidative stress?," Free Radical Research, vol. 38, no. 6, pp. 535-539, 2004.

[46] J. B. Whitfield, "Gamma glutamyl transferase," Critical Reviews in Clinical Laboratory Sciences, vol. 38, no. 4, pp. 263-355, 2001.

[47] J. H. Doroshow, "Glutathione peroxidase and oxidative stress," Toxicology Letters, vol. 82-83, pp. 395-398, 1995.

[48] P. Jovanović, L. Žorić, I. Stefanović et al., "Lactate dehydrogenase and oxidative stress activity in primary open-angle glaucoma aqueous humour," Bosnian Journal of Basic Medical Sciences, vol. 10, no. 1, pp. 83-88, 2010.

[49] S. Salzano, P. Checconi, E.-M. Hanschmann et al., "Linkage of inflammation and oxidative stress via release of glutathionylated peroxiredoxin-2, which acts as a danger signal," Proceedings of the National Academy of Sciences, vol. 111, no. 33, pp. 12157-12162, 2014.

[50] M. Abd AL Majeed, E. Y. A. AL-Sultan, and A. A. K. Abass, "Toxic effects of low concentration of cyanotoxin (microcystin-LR) on mice and study of protective efficacy of the antioxidants vitamins (C\&E) and Capparis spinosa L. root extract," Journal of Natural Sciences Research, vol. 6, pp. 34-42, 2016.

[51] A. Abdeen, M. Aboubakr, D. Elgazzar et al., "Rosuvastatin attenuates piroxicam-mediated gastric ulceration and hepatorenal toxicity in rats," Biomedicine \& Pharmacotherapy, vol. 110, pp. 895-905, 2019.

[52] A. Abdeen, A. Abdelkader, M. Abdo, G. Wareth, M. Aboubakr, and L. Aleya, "Protective effect of cinnamon against acetaminophen-mediated cellular damage and apoptosis in renal tissue," Environmental Science and Pollution Research, vol. 26, no. 1, pp. 240-249, 2019.

[53] R. K. Bhardwaj, H. Glaeser, L. Becquemont, U. Klotz, S. K. Gupta, and M. F. Fromm, "Piperine, a major constituent of black pepper, inhibits human P-glycoprotein and CYP3A4," The Journal of Pharmacology and Experimental Therapeutics, vol. 302, no. 2, pp. 645-650, 2002.

[54] Z. H. Jin, W. Qiu, H. Liu, X. H. Jiang, and L. Wang, "Enhancement of oral bioavailability and immune response of ginsenoside Rh2 by co-administration with piperine," Chinese Journal of Natural Medicines, vol. 16, no. 2, pp. 143-149, 2018. 


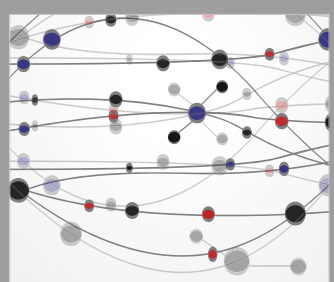

The Scientific World Journal
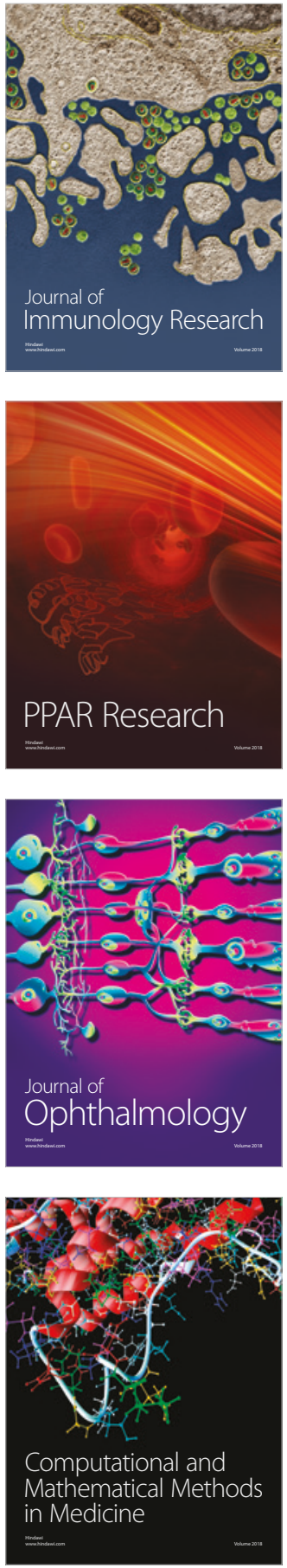

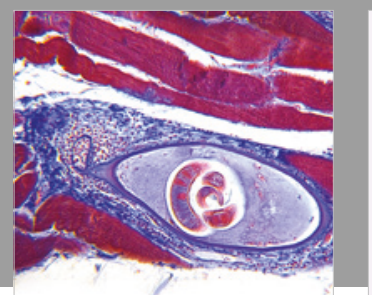

Gastroenterology Research and Practice

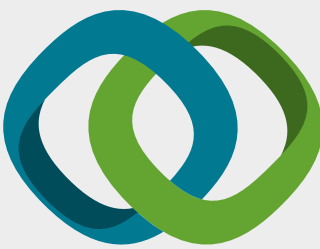

\section{Hindawi}

Submit your manuscripts at

www.hindawi.com
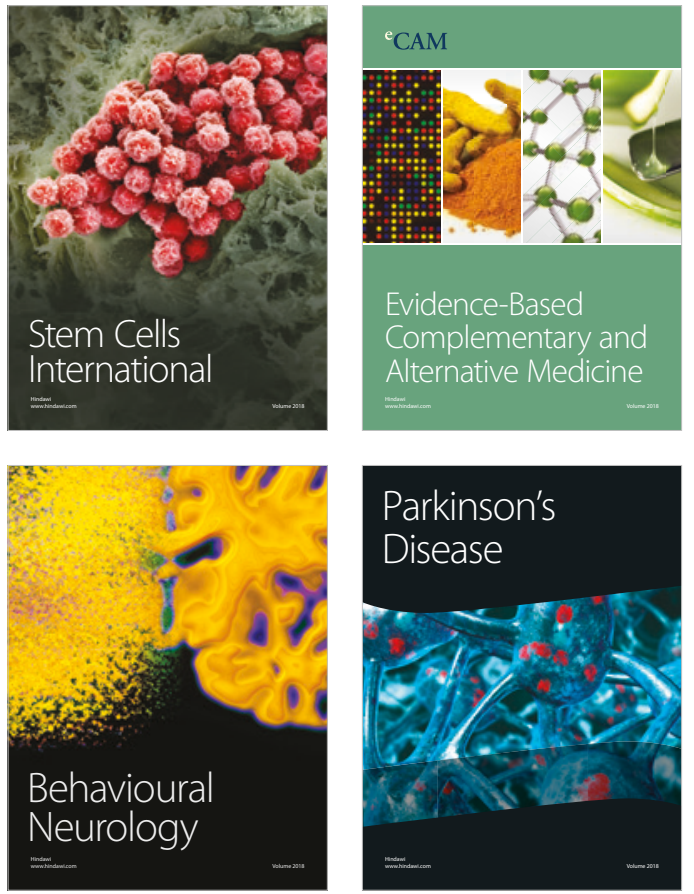

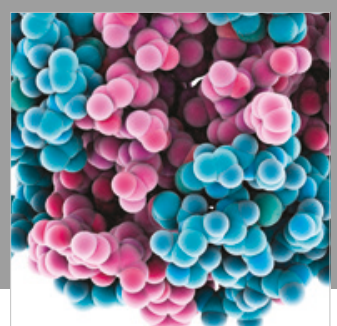

ournal of

Diabetes Research

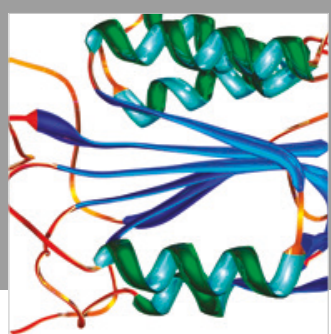

Disease Markers
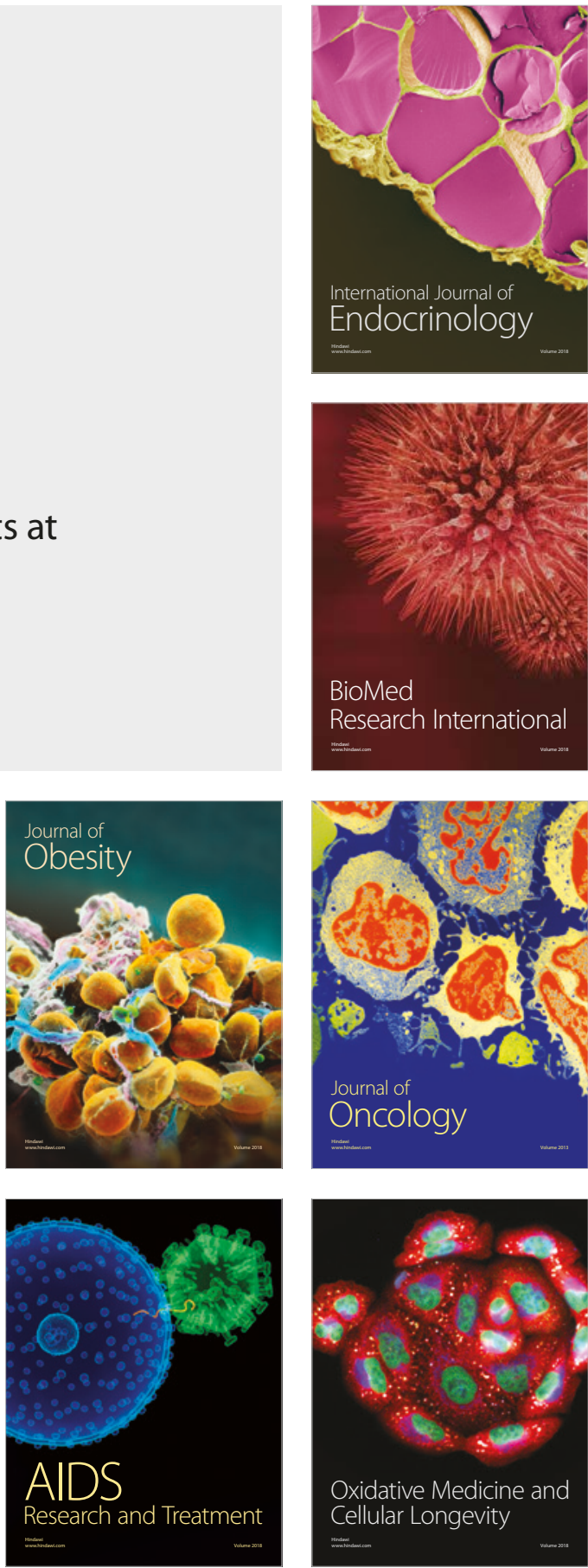